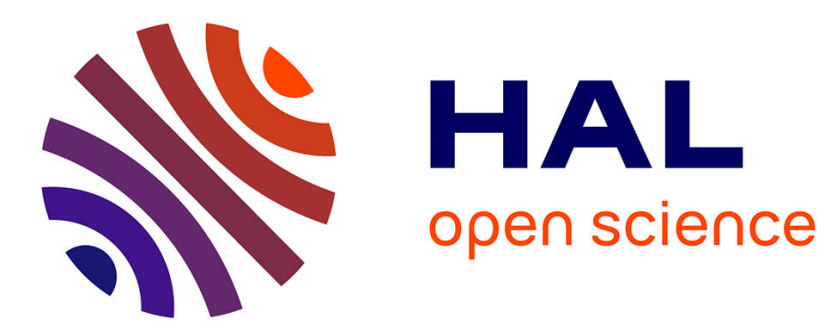

\title{
Laser optoacoustic spectroscopy : figures of merit, an up-to-date approach
}

\author{
S. Egerev, L. Lyamshev, A. Pashin
}

\section{To cite this version:}

S. Egerev, L. Lyamshev, A. Pashin. Laser optoacoustic spectroscopy: figures of merit, an up-to-date approach. Journal de Physique IV Proceedings, 1994, 04 (C7), pp.C7-425-C7-428. 10.1051/jp4:19947100 . jpa-00253152

\section{HAL Id: jpa-00253152 https://hal.science/jpa-00253152}

Submitted on 1 Jan 1994

HAL is a multi-disciplinary open access archive for the deposit and dissemination of scientific research documents, whether they are published or not. The documents may come from teaching and research institutions in France or abroad, or from public or private research centers.
L'archive ouverte pluridisciplinaire HAL, est destinée au dépôt et à la diffusion de documents scientifiques de niveau recherche, publiés ou non, émanant des établissements d'enseignement et de recherche français ou étrangers, des laboratoires publics ou privés. 


\title{
Laser optoacoustic spectroscopy: figures of merit, an up-to-date approach
}

\author{
S.V. Egerev, L.M. Lyamshev and A.E. Pashin \\ N.N. Andreev Acoustics Institute, 4 Shvernika Str., 117036 Moscow, Russia
}

\begin{abstract}
Optoacoustic (OA) detection apparatus can be used for analysis of various optical absorbers in very dilute concentrations. Parameters of pulse OA spectroscopy as applied to highly transparent samples are derived allowing to compare the potentialities to these of conventional absorption methods. We consider a frequently used OA device based upon linear thermoelastic OA conversion. Practice of both amplitude and dynamic versions of $\mathrm{OA}$ method are discussed. The role of pure solvent contribution is revealed. Useful estimates are provided.
\end{abstract}

\section{INTRODUCTION}

The term "figures of merit", as applied to optoacoustic (OA) liquid spectroscopy with direct recording of the acoustic response was broadly discussed in, say, Ref [1]. It is difficult to apply here the factors controlling sensitivity in conventional absorption spectroscopy since OA spectroscopy is of zero-background type. Hence one of the subjects under discussion is the dependence of the limiting detectable concentration of the solute upon laser pulse energy. The object of the present paper is to obtain refined versions of the figures of merit which characterize the contemporary state of OA-spectroscopy and allow to compare its potentialities with these of conventional absorption methods. Furthermore, the parameters obtained correspond to the demands of IUPAC.

\section{CHOOSING AN APPROPRIATE OA SET-UP}

Parameters of an optoacoustic set-up for future use must meet certain requirements [2]. These requirements were treated in various papers. In particular, minimal detectable thermalized energy per unit length of the sample was defined, $\epsilon_{\min }=\left(\alpha_{\text {tot }} E\right)_{\min }[1]$, where $\alpha_{\text {tot }}$ is the absorptivity of the sample, $E$ is laser pulse energy. The convenience of $\epsilon_{\min }$ is that it is unaffected by dimensions of $\mathrm{OA}$ cell. However, it follows that when using $\epsilon_{\min }$ the minimal detectable value of absorptivity, $\alpha_{\text {tot }}^{\text {min }}$, is inversely proportional to $E$, the latter fact being of limited applicability. For example, in Ref. [3] the detection limit of analyzed solute was derived directly from $\alpha_{\text {tot }}^{\min }$ thus defined. Yet another frequently used parameter is optoacoustic responsivity $Y$ defined as a ratio of the useful response magnitude to the specific thermalized energy. The most characteristic measuring configuration is the "lateral" one: the piezoelectric receiver is located in the near field of a cylindrical beam crossing the sample. In that case (see, for example, Ref. [4]) the following is 
valid, $Y=0.02 \bar{\nu} \Gamma /\left(r^{1 / 2} a_{0}^{3 / 2}\right)$, where $\bar{\nu}$ is transducer sensitivity, $r$ is a distance to the receiver, $a_{0}$ is the beam waist, $\Gamma$ is so-called Gruneisen coefficient depending, in turn, upon properties of the sample, $\Gamma=\beta c^{2} / c_{p}$. Here $\beta$ is the volume thermal-expansion coefficient, $c$ is sound velocity, $c_{p}$ is the specific heat. It is suggested that laser pulses of small duration $\tau_{l}$ are utilized, $c \tau_{l} \ll a_{0}$.

Further on, when one deals with different liquid samples it is useful to characterize the OA set-up with the product $\gamma=\Gamma l \epsilon_{\min }$ [5] which takes into account parameters of the sample and OA cell length $l$ as well. Hence the problem is to minimize $\gamma$.

We start from the point that it is not sufficient to characterize the method by any one parameter solely. On the contrary, a set of parameters is proposed.

To our opinion, the initial set-up design assumes the account of the contribution of pure solvent. OA spectroscopy is mainly used for diluted solutions investigation. Hence the solvent contribution is to be treated separately, as a starting position. Let us put down the total sample absorptivity in the form

$$
\alpha_{\text {tot }}=\alpha+\epsilon(\lambda) C,
$$

where $\alpha$ is the absorptivity of the solvent, $\epsilon(\lambda)$ is molar absorptivity of the solute to be investigated depending upon the beam wave length $\lambda, C$ is its concentration. It appears that the previously introduced $\epsilon_{\min }$ makes the sense provided that it corresponds to the first term in the right side of the equation thus describing response of the pure solvent. We restrict ourselves to the amplitude version of OA-spectroscopy at this stage. With respect to the previously introduced variables optoacoustic spectroscopy equation for receiver output amplitude can be put down as follows:

$$
U=N \epsilon(\lambda) C E Y+N \alpha E Y+\sqrt{N} \cdot u_{N},
$$

where $u_{N}$ is output noise level of the receiver and $N$ is the number of shots to be averaged. Here the second addend represents the background contribution of the pure solvent. With $C \rightarrow 0$ Eq.(1) yields a simple relation for limiting detectable absorptivity of the solvent:

$$
\alpha_{\min }=u_{N} /(E Y \sqrt{N})
$$

The value $\alpha_{\min }$ so defined is truly inversely proportional to pulse energy. It can not be used for immediate estimation of the detection limit of the solute. However it remains a very useful parameter (as it will be shown) and can be estimated as follows. Simple calculations yield $Y=1.5 \cdot 10^{8}$ $\mu \mathrm{V} \cdot \mathrm{cm} / \mathrm{J}$ in the case of water sample and typical values $a_{0}=0.1 \mathrm{~cm}, r=1 \mathrm{~cm}, \bar{\nu}=460 \mu \mathrm{V} / \mathrm{Pa}$. If energy deposits correspond to thermoelastic regime values, namely, $E=0.01 \mathrm{~J}$, we now obtain with $N=100$ and $u_{N} \sim 7 \mu \mathrm{V}$ that $\alpha_{\min }=10^{-7} \mathrm{~cm}^{-1}$.

\section{STAGE OF MEASUREMENT. AMPLITUDE VERSION}

One of analytical figures of merit, the limiting concentration of the solute $C_{\min , P}$ which can be detected within the probability $P$ is introduced as follows according to IUPAC rules:

$$
C_{\min , P}=\frac{t_{P, f}\left[S_{a}+\bar{C} S_{b}\right]}{b+t_{P, f} S_{b}},
$$

where $t_{P, f}$ is Student's coefficient, $a$ and $b$ are the coefficients of the regression curve for analytical signal magnitude $U$ obtained in the course of calibration procedures over $m$ standard solutions,

$$
U=a+b C,
$$

$f$ is the number of degrees of freedom, $f=m-2$, and $S_{a}$ and $S_{b}$ are absolute errors of $a$ and $b$ evaluation, respectively, 


$$
\bar{C}=\left[\left(\Sigma_{1}^{n} U / n\right)-a\right] / b,
$$

and $n$ is the number of measurement events. Expressions for $a$ and $b$ can be derived directly from Eq.(1). Comparing Eq.(1) and Eq.(3) we find a simplified expression for $S_{\mathrm{a}}$ :

$$
S_{a}=N E Y \sqrt{\alpha_{\min }^{2}+S_{\alpha}^{2}}
$$

where $S_{\alpha}$ is the absolute error of pure solvent response compensation. This variable contributes significantly to the total error. For typical relative error of the apparatus of 0.01 we can put $S_{\alpha} \sim 10^{-6} \mathrm{~cm}^{-1}$. The previously defined value $\alpha_{\min }$ competes with $S_{\alpha}$. It follows from Eq.(2) and Eq.(4) that the optimal set-up assumes the fulfillment of the condition $\alpha_{\min } \ll S_{\alpha}$. For this case ignoring $S_{b}$ and substituting Eq.(4) into Eq.(2) one obtains limiting detectable concentration value, independent of $E, \bar{\nu}$ and $N$ :

$$
C_{\min , P}=t_{P, f} \cdot S_{\alpha} / \epsilon(\lambda) .
$$

In our experiments test solutions of fucshine were irradiated by the second harmonics of YAG$\mathrm{Nd}^{3+}$ laser pulse $(\lambda=535 \mathrm{~nm}, \epsilon(\lambda)=0.073 \mathrm{~mL} \cdot \mathrm{cm} / \mu \mathrm{g})$. With axisymmetric $\mathrm{OA}$ cell having a piezoceramic cylindrical receiver, providing $Y=1.5 \cdot 10^{8} \mu \mathrm{V} \cdot \mathrm{cm} / \mathrm{J}$ and in view of $P=0.99$ and $t_{P, f}=5.8$ we get $\alpha_{\min } \sim 10^{-7} \mathrm{~cm}^{-1}$ and $C_{\min , P}=0.1 \mu \mathrm{g} / \mathrm{L}$.

\section{STAGE OF MEASUREMENT. DYNAMIC CONCEPT}

Despite of the spread of the methods of amplitude OA diagnostics the amount of information on the specimen under study that these methods allow one to obtain is rather limited. The concept of laser dynamic optoacoustic spectroscopy was discussed in Ref. [6]. It assumes adequate frequency- or time-domain resolution of signals and the extraction of the additional information offered by the moment of the signal arrival, signal form, the profile of the rising front, the relationship among the amplitudes of the phases of its fine structure, etc. Dynamic approach is useful, for example, if the presence of some impurity gives rise to some additional mechanism of opto-acoustic conversion.

The following is a simplified example of how we use spectral processing for spectroscopy parameters enhancement. Let us consider the microinhomogeneous liquid sample having a small amount of immersed particles of radius $R$. The limiting numeric concentration of the suspension $C_{m i n, P}^{*}$ can be estimated as follows. An auxiliary mechanism of opto-acoustic conversion connected with heat transfer from particles to solvent causes an increase of the low frequency constituents [7], i.e. exhibits itself in the frequency range $\left(0,2 \pi / \tau_{R}\right)$, where $\tau_{R} \sim R^{2} / \chi$ is the time of temperature equalization in the particle, $\chi$ is temperature conductivity. We can put now for the output signal amplitude instead of Eq.(1) with respect to these frequency changes

$$
U \sim(N / \pi) \int\left|u_{\omega, P}\right| d \omega+(N / \pi) \int\left|u_{\omega, S}\right| d \omega+\sqrt{N} \cdot u_{N}^{*}
$$

Here $u_{\omega, P}$ is voltage response spectrum induced by opto-acoustic conversion due to heat migration from particles' ensemble, $u_{\omega, S}$ is voltage response spectrum corresponding to direct heating of the pure solvent and noise level $u_{N}^{*}$ corresponds now to the frequency range chosen. In the case of instantaneous perception of the particle heat by the surrounding liquid and with respect to optical and thermodynamical properties of the particles [8] the following is valid:

$$
\left|u_{\omega, P}\right|=k_{\mathrm{a}} \pi R^{2} C^{*} E Y^{*} \tau_{R} \sqrt{\frac{\omega \tau_{R}}{1+\omega \tau_{R}}} .
$$


Here $Y^{*}=\bar{\nu} \beta c^{1 / 2} /\left(\pi^{1 / 2} c_{p} r^{1 / 2} \tau_{R}^{3 / 2}\right), k_{a}$ is so-called absorption efficiency factor of a particle [9]. The low frequency spectrum of the solvent response obeys $u_{\omega, S} \propto \sqrt{\omega \tau_{R}}$.

Upon integrating Eq.(5) over the indicated frequency range that corresponds to simple low-frequency filtration we obtain for the coefficients $a$ and $b$ in Eq.(3)

$$
a=\alpha N E Y^{*}+\sqrt{N} \cdot u_{N}^{*}, \quad b=k_{a} \pi R^{2} N E Y^{*}
$$

We can now put for the absolute error $S_{a}$ in the way similar to Eq.(4)

$$
S_{\alpha}=N E Y^{*} \sqrt{N^{-1}\left(\frac{u_{N}^{*}}{E Y^{*}}\right)^{2}+S_{\alpha}^{2}}
$$

The first addend under the root sign is very close to the previously defined $\alpha_{\text {min }}^{2}$. However the value of $S_{a}$ according to Eq.(7) is about 20 times less than that of Eq.(4) for typical measurement conditions. A considerable gain in limiting detectable concentration is a consequence of the relation Eq.(7). For the optimized set-up design we finally find from Eq.(2).

$$
C_{\min , P}^{*}=t_{P, f} \cdot S_{\alpha} / k_{a} \pi R^{2}
$$

At $R=2 \cdot 10^{-4} \mathrm{~cm}, k_{a} \sim 1$ the value $C_{\min , P}^{*} \sim 10^{2} \mathrm{~cm}^{-3}$ (volume part of the suspension $10^{-9}$ ).

\section{CONCLUSION}

These results form the basis against which useful parameters of OA diagnostics can be evaluated. It is shown that laser pulse energy, the number of shots to be averaged and piezoelectric transducer sensitivity have no effect on the limiting detectable concentration if one utilizes an optimized measurement device based upon linear thermoelastic OA conversion. The parameters listed here are used at the stage of device choice and device preparation to achieve decrease of the minimal detectable absorption of the pure solvent. Furthermore, it becomes evident that revealing some additional mechanisms (non-linear ones included) of OA conversion offers new information about the sample under study. For example, OA cavitation mechanism (not to be confused with laser induced breakdown) can be used not only to detect the suspension concentration to roughly estimate particles' dimensions as well [9]. The potentialities of such high-power versions of the discussed technique can be described by some new set of parameters.

\section{ACKNOWLEDGEMENT}

This work was supported, in part, by a Soros Foundation Grant awarded by the American Physical Society. We wish to express our thanks to Dr. O.Puchenkov for useful discussions.

\section{References}

[1] Voigtman E., Jurgensen A., Winefordner J., Anal. Chem., 53 (1981) 1442-1446.

[2] Tam A.C., "Photoacoustic and Photothermal Phenomena III" (Springer, 1992) pp 447-462.

[3] Kim J.-I., Stumpe R., Klenze R., Topics in Current Chemistry 157 (1990) 129-179.

[4] Tam A.C., Patel C.K.N., Appl. Phys. Lett., 35 (1979) 843-847.

[5] Puchenkov O.V., private communication.

[6] Egerev S.V., Lyamshev L.M., Puchenkov O.V., Sov. Phys. Usp., 33 (1990) 739-762.

[7] Sawada T., Kitamory T., "Physical Acoustics" (Academic Press, 1988) XVIII pp 347-401.

[8] Egerev S.V., Pashin A.E., Acoust. Phys., 39 (1993) 43-45.

[9] Egerev S.V., Pashin A.E., Simanovskii Ya.O., "Advances in Nonlinear Acoustics"

(World Scientific, 1993) pp 436-441 